\title{
SORÇÃo E HidRólise do Herbicida Flazasulfuron ${ }^{1}$
}

\author{
Sorption and Hydrolysis of Flazasulfuron \\ OLIVEIRA, M.F. ${ }^{2}$, PRATES, H.T. ${ }^{3}$ e SANS, L.M.A. ${ }^{4}$
}

\begin{abstract}
RESUMO - O herbicida flazasulfuron vem sendo utilizado no Brasil para o controle de plantas daninhas mono e dicotiledôneas nas culturas de cana-de-açúcar e tomate. Há carência de informações sobre o seu comportamento no ambiente. O objetivo deste trabalho foi estudar a retenção do flazasulfuron em amostras de solos, superficiais e subsuperficiais, e sua hidrólise em diferentes valores de pH e temperatura. Nos ensaios de sorção e dessorção utilizou-se metodologia de batelada em equilíbrio nas concentrações de 0,07 a 5,00 $\mathrm{mg} \mathrm{L}^{-1} \mathrm{e}$ quantificação por CLAE. A hidrólise do flazasulfuron mostrou-se dependente da temperatura e do $\mathrm{pH}$, seguindo modelo de primeira ordem. A meia-vida do herbicida em solução aquosa variou de 0,76 hora a $35^{\circ} \mathrm{C} \mathrm{e} \mathrm{pH} 3$ a 167,4 horas a $25^{\circ} \mathrm{C}$ e $\mathrm{pH} 5$. O K $\mathrm{K}_{\text {f,ads }}$ variou de 1,19 $\left(1 / \mathrm{n}_{\text {ads }}=0,40\right)$ a $27,97\left(1 / \mathrm{n}_{\text {ads }}=0,90\right)$, apresentando correlação positiva e significativa com os teores de CO, silte e CTC. A adsorção do flazasulfuron foi menor nas amostras com maiores valores de $\mathrm{pH}$, indicando maior risco de lixiviação nessa condição.
\end{abstract}

Palavras-chave: sulfoniluréias, retenção, solo.

ABSTRACT-Flazasulfuron [1-(4,6-dimethoxypyrimidin-2-yl)-3-(3-trifluoromethyl-2-pyridylsulfonyl) urea] has been used in Brazil the selective control of monocotyledonous and dicotyledonous weeds grown in the sugarcane and tomato crops. There is a lack of information in the literature about flazasulfuron behavior in soils. In this study, flazasulfuron retention was measured in batch equilibration, at 1:1 soil/solution ratio for Brazilian surface and subsurface soil samples and hydrolysis at different $\mathrm{pH}$ and temperature values. Sorption and desorption experiments were carried out at concentrations of 0.07 to $5.00 \mathrm{mgL}^{-1}$ and quantification by HPLC. Hydrolysis of flazasulfuron was dependent on temperature and $\mathrm{pH}$, following a first order model. The half-life of the herbicide in aqueous solution varied from 0.76 hours at $35^{\circ} \mathrm{C}$ and $p H 3$ to 167.4 hours at $25^{\circ} \mathrm{C}$ and $p H$ 5. Sorption isotherms were nonlinear with $K_{\text {f.ads }}$ values ranging from $1.19\left(1 / n_{\text {ads }}=0.40\right)$ to $27.97\left(1 / n_{a d s}=0.90\right)$ correlated positively and significantly to organic carbon $(r=0.78)$, silt $(r=0.75)$ and CTC $(r=0.61)$. Flazasulfuron adsorption was lower in samples with higher $p H$ values, indicating a greater risk of leaching under that condition.

Key words: sulfonylureas, retention, soil.

\section{INTRODUÇÃO}

Flazasulfuron 1-(4,6-dimethoxypyrimidin2-yl)-3-(3-trifluoromethyl-2-pyridylsulphony) urea é um herbicida pertencente ao grupo das sulfoniluréias (Figura 1), registrado no Brasil para o controle de mono (incluindo ciperáceas) e dicotiledôneas nas culturas de cana-de-açúcar (Saccharum spp.) e tomate (Lycopersicum spp.) em pré ou pós-emergência inicial, conforme a cultura (Rodrigues \& Almeida, 1998). Há carência de informações sobre o comportamento dos herbicidas em solos tropicais (Racke, 1996), especialmente do flazasulfuron nos solos brasileiros.

1 Recebido para publicação em 23.6.2004 e na forma revisada em 21.4.2005.

Parte das exigências da tese de doutorado do primeiro autor.

2 Eng.-Agrônomo, Post-Doctoral Research Associate, Purdue University, West Lafayette, 47906, IN, USA. ${ }^{3}$ Químico, Pesquisador da Embrapa Milho e Sorgo; ${ }^{4}$ Eng.-Agrônomo, Pesquisador da Embrapa Milho e Sorgo. 


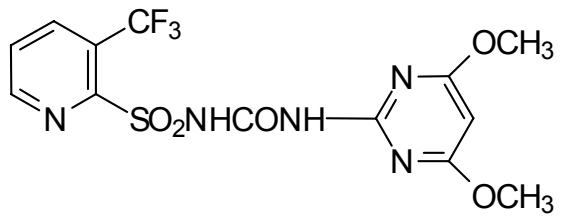

FLAZASULFURON

Solubilidade em água: $16,1 \mathrm{mg} \mathrm{L}^{-1}$ a $24^{\circ} \mathrm{C}, \mathrm{pH} 7$

$\mathrm{pH}$ : não descrito na literatura

pK : não descrito na literatura

Peso molecular: 407,3

$\mathrm{K}_{\text {ow }}: 1,08$

Pressão de vapor: $3,1 \times 10^{-8} \mathrm{mmHg}$

Figura 1 - Algumas características fisico-químicas do herbicida flazasulfuron (Hashizume, 1990).

A fim de predizer o destino do flazasulfuron no ambiente, é essencial entender o efeito das propriedades do solo na sua retenção. A retenção, especificamente a sorção, afeta os processos que regulam seu destino no ambiente, como lixiviação, volatilização, degradação química e microbiológica, absorção pelas plantas e carreamento superficial (Bailey \& White, 1970; Koskinen \& Harper, 1990).

Na solução do solo, as moléculas tendem a atingir equilíbrio entre a fase sorvida e a que permanece em solução. No entanto, a absorção pelas plantas, a eficácia e o transporte dependem em grande parte do equilíbrio entre os processos de sorção e dessorção. Geralmente, a eficiência e mobilidade dos herbicidas decrescem com o aumento da sua sorção pelos colóides do solo.

As sulfoniluréias, em geral, são compostos não-voláteis que possuem um próton ionizável no grupo amino adjacente ao grupo sulfonil, comportando-se, portanto, como ácidos fracos, com pK na faixa de 3 a 5 (Brown, 1990; Hay, 1990). Por essa razão, sua solubilidade em água a $\mathrm{pH} 7$ é aproximadamente dez vezes maior que em pH 5 (Blair \& Martin, 1988; Smith, 1995). A hidrólise dos herbicidas do grupo sulfoniluréias depende da temperatura e do $\mathrm{pH}$. O pH da solução controla a hidrólise porque a forma neutra do ligante sulfoniluréia é consideravelmente mais suscetivel à hidrólise que a forma iônica. Segundo Smith (1995), a meia-vida das sulfoniluréias chlorsulfuron, chlorimuron-ethyl, metsulfuron methyl e sulfometuron ethyl em meio aquoso a $45^{\circ} \mathrm{C}$ e pH 5,0 foi de 1,$7 ; 0,6 ; 2,1$; e 0,4 dias, enquanto em condições similares a pH 7,0 os dados de meia-vida foram de 51, 14, 33 e 6 dias, respectivamente. Bertrand et al. (2003) observaram que o herbicida flazasulfuron teve rápida degradação em álcool (metanol ou etanol) a $30{ }^{\circ} \mathrm{C}$ e que a hidrólise seguiu cinética de primeira ordem e dependente de $\mathrm{pH}$.

A sorção das sulfoniluréias varia em função do conteúdo de matéria orgânica, $\mathrm{pH}$, textura e mineralogia dos solos (Gonzales \& Ukrainczyk, 1996; Mersie \& Foy, 1985; Shea, 1986; Thirunarayanan et al., 1985; Werkheiser \& Anderson, 1996). Devido à sua alta solubilidade em água e fraca sorção nos solos com valores de $\mathrm{pH}$ neutros e alcalinos, as sulfoniluréias apresentam grande potencial de lixiviação em campo, principalmente sob altas precipitações pluviométricas (Blair \& Martin, 1988; Hay, 1990; Brown, 1990; Smith \& Aubin, 1993). Nesse caso, pode ocorrer lixiviação dos herbicidas para camadas mais profundas do solo, que, geralmente, possuem propriedades diferentes daquelas das camadas superficiais. Variações nas propriedades do solo com a profundidade podem afetar a retenção, o movimento e a degradação dos herbicidas (Felding, 1997; Harper, 1988; Jenks et al., 1998).

A sorção do chlorsulfuron e metsulfuronmethyl em solos de diferentes profundidades foi correlacionada negativamente com o $\mathrm{pH}$ do solo e positivamente com o conteúdo de matéria orgânica deste, sendo $\mathrm{o} \mathrm{pH}$ considerado fator dominante no controle da adsorção para a maioria dos solos estudados (Walker et al., 1989). Gonzales \& Ukrainczyk (1996), estudando a sorção e dessorção do nicosulfuron, verificaram correlação da sorção desse herbicida com o conteúdo de argila para os solos de Iowa, enquanto para os solos do Brasil a sorção foi correlacionada com o conteúdo de matéria orgânica. Esses autores atribuíram essa diferença na sorção às diferenças na mineralogia entre os solos de Iowa (minerais de argila 2:1 expansiveis) e os do Brasil (caulinita e óxidos de $\mathrm{Fe}$ e $\mathrm{Al}$ ).

A degradação das sulfoniluréias no solo segue a mesma dependência do $\mathrm{pH}$ observada 
em soluções tamponadas em água (Smith \& Aubin, 1993; Blair \& Martin, 1988; Stork, 1995; Smith, 1995). Em solos ácidos, a degradação ocorre preferencialmente por hidrólise, enquanto em solos com altos valores de $\mathrm{pH}$ a hidrólise se reduz drasticamente, e a degradação se deve principalmente à microbiota do solo (Smith \& Aubin, 1993; Blair \& Martin, 1988; Smith, 1995).

A sorção do flazasulfuron depende da afinidade do solo pela forma neutra, predominante quando $\mathrm{pH}<\mathrm{pK}_{\mathrm{a}}$, e forma aniônica, dominante quando $\mathrm{pH}^{>}>\mathrm{pK}_{\mathrm{a}}$. A forma neutra do flazasulfuron pode ser sorvida pela matéria orgânica, enquanto a forma aniônica pode ser sorvida pelos grupos hidroxilas protonados dos óxidos de ferro e alumínio. $\mathrm{O}$ aumento de $\mathrm{pH}$ acarreta diminuição na quantidade de flazasulfuron na forma neutra, o qual causa redução na sorção pela matéria orgânica. $\mathrm{O}$ aumento do $\mathrm{pH}$ diminui o número de sítios do solo carregados positivamente, acarretando decréscimo na sorção do flazasulfuron aniônico. Por isso, a sorção do flazasulfuron poderá diminuir com o aumento do $\mathrm{pH}$, incrementando seu potencial de lixiviação em solos cujo $\mathrm{pH}$ se aproxima da neutralidade. Como a sorção afeta a mobilidade e a degradação e, conseqüentemente, o potencial de contaminação de águas superficiais e subterrâneas, informações sobre sorção e degradação em água do flazasulfuron contribuem para melhor entendimento do seu comportamento e destino no ambiente.

Há carência de estudos que mostram o efeito das propriedades do solo sobre a sorção e dessorção do flazasulfuron no solo. O objetivo deste trabalho foi determinar a influência das propriedades de amostras de solos, superficiais e subsuperficiais, na sorção, na dessorção e na hidrólise do flazasulfuron em diferentes valores de $\mathrm{pH}$ e temperatura.

\section{MATERIAL E MÉTODOS}

Os ensaios foram conduzidos no Laboratório de Agroquímica da Embrapa Milho e Sorgo, em Sete Lagoas, MG. Todas as análises foram feitas em condições de temperatura ambiente controlada $\left(27 \pm 1^{\circ} \mathrm{C}\right)$.

\section{Ensaio de hidrólise do flazasulfuron}

Para o ensaio de hidrólise foram colocados $30 \mathrm{~mL}$ da solução de flazasulfuron $4 \mathrm{mg} \mathrm{L}^{-1}$ (aproximadamente 95\% de pureza, fornecidos pela ISK Bioscience), previamente preparada em solução $\mathrm{CaCl}_{2}$ 0,01 $\mathrm{M}$ em erlenmeyers com capacidade de $50 \mathrm{~mL}$. Os valores de $\mathrm{pH}$ foram obtidos adicionando-se pequenas quantidades de solução $\mathrm{HCl}$ 0,01 M, para o pH 3 e 5 e de solução $\mathrm{NaOH}$ 0,01 M para o pH 8. Em seguida, os erlenmeyers foram cobertos com papelalumínio e imersos num agitador com banhomaria em temperatura controlada. As temperaturas utilizadas foram de 25,30 e $35 \pm 1{ }^{\circ} \mathrm{C}$. Quando necessário, os valores de $\mathrm{pH}$ foram reajustados para os valores iniciais durante a condução do experimento. A amostragem foi feita retirando-se alíquotas de $0,5 \mathrm{~mL}$ em cada combinação temperatura/pH em intervalos de uma hora, totalizando 13 amostras por combinação, acondicionadas em tubos de polietileno com capacidade de $1 \mathrm{~mL}$. Imediatamente após amostragem, os tubos foram armazenados no freezer até injeção no cromatógrafo. Não foi observada retenção do flazasulfuron pelos tubos.

A quantificação do flazasulfuron foi feita em cromatógrafo líquido de alta eficiência (CLAE), marca Shimadzu, modelo LC 10A, com detector UV/Vis. Foi utilizada a técnica do padrão externo, com curva de calibração linear baseada na integração da área do pico construída com padrão analítico, método dos quadrados mínimos, $\mathrm{R}^{2}=99,49 \%$, AUFS de 0,002, limite de detecção e quantificação de $4 \mu \mathrm{g} \mathrm{L}^{-1}$.

Condições analíticas: a fase móvel consistiu da mistura água:acetonitrila $(1: 1)$ a $\mathrm{pH} 3,0$, ajustado com ácido ortofosfórico 0,04\%; coluna Adsorbosil C-18, marca Alltech, com 4,6 mm x $15 \mathrm{~cm}$ e diâmetro de poro de $5 \mu \mathrm{m}$; coluna à

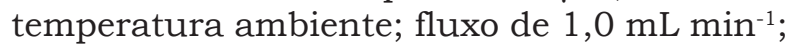
"loop" de $100 \mu \mathrm{L}$; e detector UV com comprimento de onda de $245 \mathrm{~nm}$.

O logaritmo dos valores de concentrações obtidas foram plotados em função do tempo de amostragem. Em seguida, foi ajustada equação de primeira ordem para o cálculo da constante de velocidade $\left(\mathrm{K}_{1}\right)$, meia-vida $\left(\mathrm{t}_{1 / 2}\right)$ do herbicida em solução, inclinação da equação de Arrhenius e Energia de Ativação $\left(\mathrm{E}_{\mathrm{a}}\right)$ nas diferentes combinações de $\mathrm{pH}$ e temperatura.

Planta Daninha, Viçosa-MG, v. 23, n. 1, p. 101-113, 2005 


\section{Ensaios de sorção/dessorção do flazasulfuron}

\section{Solos estudados}

O estudo de sorção e dessorção do flazasulfuron foi realizado utilizando-se amostras coletadas na camada arável $(0-15 \mathrm{~cm})$ do: 1) Latossolo Vermelho-Escuro distrófico, A moderado, textura média - LE; 2) Latossolo Roxo distrófico, A moderado, textura argilosa LRd; 3) Gley Húmico, Tb, A proeminente, textura média - GH, na Universidade de São Paulo, campus "Luiz de Queiroz" (padronizados pelo IBAMA), no município de Piracicaba-SP; e 4) Latossolo Vermelho-Escuro álico, A moderado, textura argilosa - LEr, amostrado na Embrapa Milho e Sorgo, município de Sete Lagoas - MG. Nesse último, foi aberto perfil, onde foram coletadas amostras de $15 \mathrm{em}$ $15 \mathrm{~cm}$, até a profundidade de $120 \mathrm{~cm}$. Todas as amostras foram passadas em peneira de $2 \mathrm{~mm}$, secas ao ar, sendo em seguida caracterizadas física e quimicamente, segundo metodologia proposta pela Embrapa (1997) (Tabela 1).

\section{Determinação da relação solo:solução e tempo de equilibrio}

$\mathrm{Na}$ determinação da relação solo:solução e tempo de agitação da mistura utilizou-se metodologia proposta pelo IBAMA (1996). Para isso, amostras em duplicata de 10, 5, 2,5 e $1,25 \mathrm{~g}$ dos solos em estudo foram pesadas em tubos de centrifuga e misturadas com $10 \mathrm{~mL}$ da solução de $4 \mathrm{mg} \mathrm{L}^{-1}$ do flazasulfuron, previamente dissolvidos em $\mathrm{CaCl}_{2}$ 0,01M. Após agitação por 24 horas, as amostras foram centrifugadas a $2.200 \mathrm{rpm}(921,53 \mathrm{xg})$ por 10 minutos, filtradas e quantificadas no HPLC. A relação solo:solução escolhida foi a que permitiu que 20 a $80 \%$ da quantidade do herbicida aplicada fosse sorvida pelo solo, após período de equilíbrio de 24 horas.

Escolhida a relação solo:solução, esta foi agitada por 1, 5, 9, 24 e 48 horas. Após agitação, as misturas foram centrifugadas a $2.200 \mathrm{rpm}$ por 10 minutos, filtradas e quantificadas no HPLC. O tempo para o estabelecimento do equilíbrio foi definido como o tempo entre o começo do experimento até o tempo em que a concentração do herbicida na solução permanecesse constante dentro de um intervalo de 24 horas (IBAMA, 1996).

\section{Sorção/Dessorção}

Para esses ensaios, utilizaram-se as relações solo:solução determinadas preliminarmente, que foram de 1:1 para LRd, LE e LEr e 1:8 para GH. Portanto, amostras de $10 \mathrm{~g}$ dos solos LRd, LE e LEr e de 1,25 g do solo GH foram colocadas em tubos de centrífuga, com

Tabela 1 - Principais características físicas e químicas dos solos utilizados neste estudo, sua profundidade de amostragem e sua mineralogia

\begin{tabular}{|c|c|c|c|c|c|c|c|c|c|c|c|}
\hline \multirow{3}{*}{ Classe } & \multirow{2}{*}{ Prof. } & \multicolumn{7}{|c|}{ Solo } & \multicolumn{3}{|c|}{ Mineralogia $^{1^{\prime}}$} \\
\hline & & $\mathrm{pH}$ & $\mathrm{CO}$ & Silte & Argila & $\mathrm{AgN}$ & $\mathrm{U}(\%)$ & \multirow{2}{*}{$\begin{array}{c}\text { CTC } \\
\left(\mathrm{cmol}_{\mathrm{c}} \mathrm{kg}^{-1}\right)\end{array}$} & \multirow{2}{*}{ Domi } & \multirow{2}{*}{ Outr } & \multirow{2}{*}{ Textura ${ }^{2}$} \\
\hline & $(\mathrm{cm})$ & & \multicolumn{5}{|c|}{$(\%)$} & & & & \\
\hline LE & $0-15$ & 6,7 & 0,71 & 04 & 22 & 16 & 11,0 & 6,98 & $\mathrm{C}, \mathrm{H}$ & $\mathrm{G}$ & FAA \\
\hline LRd & $0-15$ & 5,1 & 1,55 & 13 & 68 & 36 & 27,0 & 11,07 & $\mathrm{C}, \mathrm{H}$ & G & MA \\
\hline $\mathrm{GH}$ & $0-15$ & 4,1 & 5,06 & 45 & 44 & 26 & 43,6 & 21,83 & -3" & 3 & AS \\
\hline LEr1 & $0-15$ & 5,8 & 2,29 & 18 & 73 & 13 & 33,9 & 9,16 & $\mathrm{C}, \mathrm{H}$ & $\mathrm{G}$ & MA \\
\hline LEr2 & $15-30$ & 5,4 & 1,73 & 14 & 77 & 19 & 34,0 & 8,08 & $\mathrm{C}, \mathrm{H}$ & G & MA \\
\hline LEr3 & $30-45$ & 5,0 & 1,47 & 10 & 81 & 08 & 34,0 & 8,58 & $\mathrm{C}, \mathrm{H}$ & G & MA \\
\hline LEr4 & $45-60$ & 5,2 & 1,43 & 03 & 87 & 14 & 33,4 & 7,34 & $\mathrm{C}, \mathrm{H}$ & $\mathrm{G}$ & MA \\
\hline LEr5 & $60-75$ & 5,1 & 1,05 & 07 & 83 & 21 & 32,1 & 7,23 & $\mathrm{C}, \mathrm{H}$ & G & MA \\
\hline LEr6 & $75-90$ & 5,1 & 1,03 & 14 & 77 & 12 & 31,4 & 6,87 & $\mathrm{C}, \mathrm{H}$ & G & MA \\
\hline LEr7 & $90-105$ & 5,2 & 0,95 & 09 & 81 & 06 & 31,3 & 6,52 & $\mathrm{C}, \mathrm{H}$ & G & MA \\
\hline LEr8 & $105-120$ & 5,1 & 1,02 & 03 & 87 & 10 & 31,5 & 6,36 & $\mathrm{C}, \mathrm{G}$ & $\mathrm{H}$ & MA \\
\hline
\end{tabular}

${ }^{1 /}$ C: caulinita; G: gibbsita; H: hematita; ${ }^{2 /}$ AS: argila siltosa; FAA: franco-argilo-arenoso; MA; muito argiloso; Prof.: profundidade; CO: carbono orgânico; AgN: argila natural; U(\%): umidade (peso) a 0,3 bar; CTC: capacidade de troca catiônica; ${ }^{3 /}$ sem referências. 
capacacidade de $60 \mathrm{~mL}$. Nesses tubos foram adicionados $10 \mathrm{~mL}$ da solução de $0,01 \mathrm{M}$ de $\mathrm{CaCl}_{2}$ contendo flazasulfuron nas concentrações de $0 ; 0,07 ; 0,2 ; 0,8 ; 1,5 ; 3,0 ;$ e $5,0 \mathrm{mg} \mathrm{L}^{-1}$, nos solos LRd, LE, LEr e GH. Todas as combinações foram repetidas duas vezes. As soluções-padrão de herbicida em cloreto de cálcio foram preparadas sempre 24 horas antes de sua utilização e, nesse período, armazenadas em geladeira $\left(5^{\circ} \mathrm{C}\right)$.

As misturas foram agitadas por 24 horas a $200 \mathrm{rpm}$, a $27 \pm 1^{\circ} \mathrm{C}$. Posteriormente, os tubos foram centrifugados a $2.200 \mathrm{rpm}$, por 10 minutos. O sobrenadante recolhido foi passado em filtro de membrana PVDF de 0,45 $\mu \mathrm{m}$. A retenção do herbicida pelas membranas dos filtros foi menor que $1 \%$.

Para o ensaio da primeira dessorção do flazasulfuron, completou-se o volume dos tubos utilizados no ensaio de adsorção para $10 \mathrm{~mL}$ com solução de $0,01 \mathrm{M}$ de $\mathrm{CaCl}_{2}$, considerando a solução retida pelo solo no ensaio de sorção. Completado o volume, os tubos foram novamente levados para agitação por 24 horas a $200 \mathrm{rpm}$, a $27 \pm 1{ }^{\circ} \mathrm{C}$, e em seguida centrifugados a $2.200 \mathrm{rpm}$ por 10 minutos. Após essa etapa, o sobrenadante foi filtrado em membrana de PVDF de 0,45 $\mu \mathrm{m}$. Tanto as amostras do ensaio de sorção quanto as de dessorção foram armazenadas em freezer, imediatamente após centrifugação, até o momento da injeção no cromatógrafo.

A quantificação e detecção do flazasulfuron foram feitas segundo metodologia descrita para o Ensaio de Hidrólise do Flazasulfuron.

$\mathrm{O}$ pH do sobrenadante, tanto no ensaio de sorção quanto no de dessorção, foi medido antes da injeção das amostras no cromatógrafo, utilizando-se peagômetro marca Digimed (modelo DM 21).

A concentração sorvida (Cs, $\mathrm{mg} \mathrm{kg}^{-1}$ solo), descontada a concentração obtida no branco, foi calculada por diferença entre a concentração das soluções inicial ou adicionada $(\mathrm{Ci}$, $\mathrm{mg} \mathrm{L}^{-1}$ ) e a concentração de equilíbrio (Ce, $\left.\mathrm{mg} \mathrm{L}^{-1}\right): \mathrm{Cs}=(\mathrm{Ci}-\mathrm{Ce})(\mathrm{V} / \mathrm{M})$, em que $\mathrm{V}$ é o volume de solução (L) e M a quantidade de solo (kg). A concentração retida pelo solo na primeira dessorção, ou seja, a concentração do flazasulfuron não dessorvida após 24 horas (Xd), foi obtida por diferença entre a concentração retida pelo solo no ensaio de adsorção e a obtida no ensaio de dessorção, utilizando a equação: $\mathrm{Xd}=[(\mathrm{Cs} \mathrm{M}+$ Ce Vret $)-\mathrm{Cd}$ Vd) $] \mathrm{M}^{-1}$, em que Vret é o volume que permaneceu retido no solo após ensaio de adsorção, $\mathrm{Cd}$ é a concentração do herbicida na solução sobrenadante no ensaio de dessorção e Vd é a soma de Vret com volume de solução $\mathrm{CaCl}_{2}$ acrescentado no ensaio de dessorção para completar $10 \mathrm{~mL}$.

Ajustou-se isoterma de Freundlich com as concentrações sorvidas e em solução: $\mathrm{X}=\mathrm{K}_{\mathrm{f}} \mathrm{X}$ $\mathrm{C}^{1 / \mathrm{n}}$, em que X é a quantidade sorvida $\left(\mathrm{mg} \mathrm{kg}^{-1}\right)$, $\mathrm{K}_{\mathrm{f}}$ e $\mathrm{n}$ são constantes para cada sistema solo: solução e C é a concentração de equilíbrio $\left(\mathrm{mg} \mathrm{L}^{-1}\right)$. Regressão não-linear foi utilizada para ajustar a equação de Freundlich para o cálculo dos parâmetros $\mathrm{K}_{\mathrm{f} \text {,ads }}$ e $1 / \mathrm{n}_{\mathrm{ads}}$ (sorção) e $\mathrm{K}_{\mathrm{f}, \mathrm{des}}$ e $1 / \mathrm{n}_{\text {des }}$ (dessorção), em todas as combinações solo:solução. A constante de sorção normalizada para carbono orgânico $\left(\mathrm{K}_{\mathrm{foc}}\right)$ foi calculada utilizando-se $\mathrm{K}_{\mathrm{f} \text {,ads }}$ na expressão: $\mathrm{K}_{\mathrm{foc}}=\left(\mathrm{K}_{\mathrm{f}, \text { ads }} / \% \mathrm{CO}\right) \times 100$; em seguida, procedeu-se â análise de Correlação de Pearson.

\section{RESULTADOS E DISCUSSÃO}

\section{Ensaio de hidrólise}

Na Figura 2, a ocorrência da reação de hidrólise se verifica pela redução na concentração do herbicida flazasulfuron com aumento da temperatura e redução no valor de $\mathrm{pH}$. Os maiores valores de concentração do flazasulfuron foram obtidos na combinação da temperatura de $25{ }^{\circ} \mathrm{C}$ e $\mathrm{pH}$ 8. Por outro lado, menores valores de concentração foram obtidos a pH 3 na temperatura de $35^{\circ} \mathrm{C}$. Essa observação está de acordo com diversos autores (Brown, 1990; Hay, 1990; Vicari et al., 1996) e com Sabadie (1990, 1991), que verificaram hidrólise do metsulfuron-metil e do chlorsulfuron com o aumento da temperatura e a redução do pH.

Considerando apenas o efeito da temperatura sobre a concentração do herbicida, verifica-se que houve redução da concentração com o aumento da temperatura da solução, em todos os níveis de $\mathrm{pH}$.

Avaliando o efeito do $\mathrm{pH}$ isoladamente, observa-se que em todas as temperaturas testadas a concentração obtida foi menor com a redução do $\mathrm{pH}$, indicando hidrólise mais 
intensa do herbicida nessa condição. Isso pode ser evidenciado pela constante de velocidade da reação de hidrólise (Tabela 2), em que os maiores valores foram obtidos a $\mathrm{pH} 3$, indicando maior velocidade da reação de hidrólise. Smith \& Aubin (1993) verificaram menores taxas de hidrólise do amidosulfuron a $\mathrm{pH} 7 \mathrm{e}$ 9, comparadas com as obtidas a pH 5, em todas as temperaturas estudadas.

O efeito do pH na redução da concentração do flazasulfuron foi mais acentuado do que aquele provocado pela temperatura, o que pode

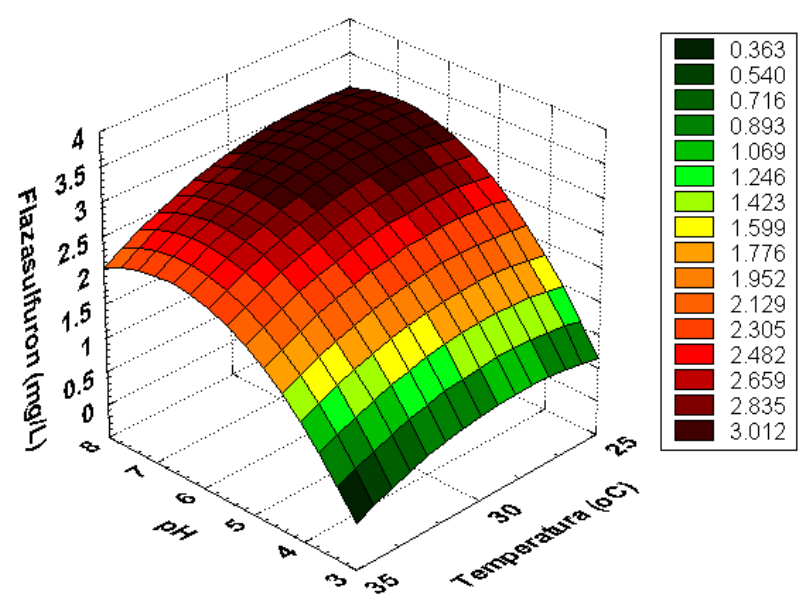

$\hat{Y}=-15,848+2,418 p H+0,792 T E-0,157 \mathrm{pH}^{2}-0,009 p H T E-0,014 T^{2}$

Figura 2 - Hidrólise do flazasulfuron em diferentes valores de $\mathrm{pH}$ e temperatura. ser visualizado pelo maior coeficiente multiplicador na equação $\left(-0,157 \mathrm{pH}^{2}\right)$ (Figura 2), ilustrado pela inclinação acentuada da curva. $\mathrm{O}$ pH da solução controla a taxa de hidrólise porque a forma neutra do ligante sulfoniluréia é consideravelmente mais suscetível à hidrólise que a forma iônica. Esses resultados estão de acordo com aqueles obtidos por diversos autores (Smith, 1995; Meyer \& Thurman, 1995; Bertrand et al., 2003; Sarmah et al., 2004), que também observaram hidrólise de outras sulfoniluréias, através do rompimento da ligação uréia, em condições ácidas. Por outro lado, em condições alcalinas, a hidrólise dos herbicidas sulfoniluréias tende a ocorrer pela ruptura do grupo metoxi (Smith, 1995). Esses resultados estão de acordo com os obtidos por Bertrand et al. (2003), os quais descreveram mínimas taxas de degradação do flazasulfuron em valores de $\mathrm{pH}$ 6-9. Ainda concordando com os resultados obtidos, esses autores descreveram rápida degradação sob condições ácidas $(\mathrm{pH} \leq 6)$ e principalmente em meios alcalinos ( $\mathrm{pH} \geq 9$ ), com taxas de degradação descritas pela cinética de primeira ordem.

A meia-vida do flazasulfuron decresceu com o aumento da temperatura, em todos os valores de $\mathrm{pH}$ (Tabela 2). Nesta tabela, os valores de meia-vida acima de 13 horas foram obtidos por extrapolação. Os valores de meia-vida do herbicida variaram de 0,67 a 167,39 horas, sendo maior no valor de $\mathrm{pH} 5$ a $25{ }^{\circ} \mathrm{C}$. A energia de ativação da reação de

Tabela 2 - Valores da constante de velocidade da reação, meia-vida, inclinação da equação de Arrhenius e energia de ativação para reação de hidrólise do flazasulfuron a diferentes valores de $\mathrm{pH}$ e temperatura

\begin{tabular}{|c|c|c|c|c|c|}
\hline \multirow[t]{2}{*}{$\mathrm{pH}$} & Temperatura & $\begin{array}{c}\text { Constantes de } \\
\text { velocidade da reação }\end{array}$ & Meia-vida $\left(t_{1 / 2}\right)$ & $\begin{array}{c}\text { Inclinação da equação } \\
\text { Arrhenius } \\
\end{array}$ & $\begin{array}{c}\text { Energia de } \\
\text { ativação }\end{array}$ \\
\hline & $\left({ }^{\circ} \mathrm{C}\right)$ & $\left(\mathrm{K}_{1} \times 10^{-3} \mathrm{~min}^{-1}\right)$ & (horas) & $\left(\mathrm{K}^{-1}\right)$ & $\left(\mathrm{KJ} \mathrm{mol}^{-1}\right)$ \\
\hline \multirow{3}{*}{3} & 25 & 5,07 & 2,28 & \multirow{3}{*}{$-4.932,9$} & \multirow{3}{*}{94,45} \\
\hline & 30 & 7,83 & 1,48 & & \\
\hline & 35 & 17,50 & 0,67 & & \\
\hline \multirow{3}{*}{5} & 25 & 0,07 & 167,39 & \multirow{3}{*}{$-11.251,0$} & \multirow{3}{*}{215,42} \\
\hline & 30 & 0,69 & 16,72 & & \\
\hline & 35 & 1,15 & 10,03 & & \\
\hline \multirow{3}{*}{8} & 25 & - & - & \multirow{3}{*}{$-9.759,4$} & \multirow{3}{*}{186,86} \\
\hline & 30 & 0,14 & 83,63 & & \\
\hline & 35 & 0,46 & 25,07 & & \\
\hline
\end{tabular}


hidrólise do flazasulfuron aumentou com o valor de $\mathrm{pH}$, sendo obtido maior valor no $\mathrm{pH} 5$, seguindo a mesma tendência da inclinação da equação de Arrhenius. Sabadie (1991) observou redução na energia de ativação na reação de hidrólise do chlorsulfuron em soluções com menores valores de $\mathrm{pH}$.

\section{Sorção/dessorção do flazasulfuron}

Os parâmetros da isoterma de Freundlich ajustada para sorção do flazasulfuron nos diferentes solos encontram-se na Tabela 3. Os valores de $\mathrm{K}_{\mathrm{f} \text {,ads }}$ variaram de 1,19 a 27,97 , sendo os maiores obtidos para os horizontes superficiais dos solos GH e LEr1, os quais possuem os maiores teores de carbono orgânico (Tabela 1). Em geral, os valores obtidos neste estudo são superiores aos descritos por Oliveira Jr. et al. (2000) para o sulfometuronmetil, nicosulfuron e metsulfuron-metil em solos brasileiros.

Apesar de o solo GH apresentar teor de carbono orgânico 2,2 vezes maior que o do $\mathrm{LEr} 1$, verifica-se que os valores de $\mathrm{K}_{\mathrm{f}, \mathrm{ads}}$ são muito próximos, diferindo apenas em 3,7 unidades. Como a relação solo:solução utilizada para o GH foi de 1:8, isso demonstra a grande afinidade do flazasulfuron pelo $\mathrm{CO}$, indicando que essa característica foi determinante na sorção desse herbicida (Tabela 4). Malik \& Drennan (1989) observaram aumentos na sorção do fluridone ao utilizarem a relação 1:1, comparada com a relação 1:5. Vários autores (Oliveira, 1995; Rhodes et al., 1970; Roowell, 1994; Schroeder, 1994) também constataram que aumentos nos teores de $\mathrm{CO}$ resultam em aumentos na afinidade dos pesticidas pelo solo. Assim como observado por Werkheiser \& Anderson (1996), verifica-se que, à exceção do $\mathrm{LE}$, para solos com $\mathrm{pH}$ semelhante, os maiores coeficientes de sorção para o flazasulfuron foram obtidos nos solos com maiores teores de CO.

Os valores de $\mathrm{K}_{\mathrm{f} \text {,ads }}$ obtidos para o flazasulfuron foram superiores aos observados para outras sulfoniluréias (Thirunarayanan et al., 1985; Gonzales \& Ukrainczyk, 1996; Werkheiser \& Anderson, 1996; Ukrainczyk \& Ajwa, 1996). As isotermas de sorção do presente trabalho foram obtidas de solos com altos teores de argila, valores de $\mathrm{pH}$ baixos e próximos ao $\mathrm{pK}_{\mathrm{a}}$ das sulfoniluréias (3 a 5) e estreita relação solo:solução. Essas condições tendem a aumentar a interação entre as moléculas do herbicida e os colóides do solo, especialmente pelo CO (Berglof et al., 2003).

Na Tabela 4, observa-se que, além do CO, o $\mathrm{K}_{\mathrm{f} \text {,ads }}$ correlacionou-se positivamente com os teores de silte, CTC e umidade quando todos os solos foram analisados em conjunto. Entretanto, quando a análise de correlação foi realizada somente com os Latossolos, ou seja, sem o GH (dado não apresentado), a correlação com o teor de silte e CTC não apresentou efeito

Tabela 3 - Parâmetros da isoterma de Freundlich e os coeficientes de determinação $\left(\mathrm{R}^{2}\right)$ das isotermas de sorção e dessorção do flazasulfuron nos solos estudados

\begin{tabular}{|c|c|c|c|c|c|c|c|c|c|c|}
\hline \multicolumn{6}{|c|}{ Sorção } & \multicolumn{5}{|c|}{ Dessorção } \\
\hline Solo & $\mathrm{pH}^{1 /}$ & $1 / \mathrm{n}_{\mathrm{ads}}$ & $\mathrm{K}_{\mathrm{f}, \mathrm{ads}}$ & $\mathrm{K}_{\text {foc }}$ & $\mathrm{R}^{2}$ & $\mathrm{pH}^{1 /}$ & $1 / \mathrm{n}_{\mathrm{des}}$ & $\mathrm{K}_{\mathrm{f}, \mathrm{des}}$ & $\mathrm{R}^{2}$ & $\mathrm{n}_{\mathrm{des}} / \mathrm{n}_{\mathrm{ads}}$ \\
\hline LE & 6,6 & 0,40 & 1,19 & 168,0 & 90,4 & 6,2 & 0,91 & 2,53 & 97,7 & 0,44 \\
\hline LRd & 4,7 & 0,74 & 4,19 & 270,5 & 98,8 & 4,5 & 0,89 & 8,52 & 96,9 & 0,83 \\
\hline GH & 4,5 & 0,90 & 27,97 & 552,4 & 98,0 & 4,6 & 0,70 & 37,51 & 85,8 & 1,29 \\
\hline LEr1 & 5,3 & 1,02 & 24,29 & $1.060,7$ & 97,2 & 4,4 & 0,77 & 24,86 & 98,9 & 1,32 \\
\hline LEr2 & 4,4 & 0,82 & 18,45 & $1.066,5$ & 97,0 & 4,3 & 0,77 & 28,61 & 99,1 & 1,06 \\
\hline LEr3 & 4,0 & 0,63 & 16,16 & $1.099,1$ & 98,4 & 4,0 & 0,50 & 16,65 & 98,8 & 1,26 \\
\hline LEr4 & 4,1 & 0,42 & 11,26 & 787,6 & 99,9 & 4,2 & 0,46 & 16,77 & 99,9 & 0,91 \\
\hline LEr5 & 4,3 & 0,65 & 14,06 & $1.339,1$ & 96,8 & 4,0 & 0,58 & 17,85 & 99,0 & 1,12 \\
\hline LEr6 & 4,3 & 0,59 & 11,56 & $1.122,3$ & 88,9 & 4,0 & 0,58 & 14,78 & 98,9 & 1,02 \\
\hline LEr7 & 4,2 & 0,64 & 10,13 & $1.066,3$ & 97,9 & 4,3 & 0,59 & 12,53 & 95,9 & 1,08 \\
\hline LEr8 & 4,3 & 0,65 & 8,26 & 809,8 & 97,7 & 4,2 & 1,07 & 24,81 & 95,5 & 0,61 \\
\hline
\end{tabular}

${ }^{1 /} \mathrm{pH}$ do sobrenadante. 
Tabela 4 - Matriz de Correlação de Pearson (r) entre as variáveis de sorção do flazasulfuron e propriedades do solo

\begin{tabular}{|c|c|c|c|c|c|c|}
\hline \multirow{2}{*}{ Todos os Solos } & $\mathrm{CO}$ & Silte & Argila & \multirow{2}{*}{$\frac{\mathrm{CTC}}{\left(\mathrm{cmol}_{\mathrm{c}} \mathrm{kg}^{-1}\right)}$} & \multirow{2}{*}{$\mathrm{U}(\% \mathrm{P})$} & \multirow{2}{*}{$\mathrm{pH}^{1 /}$} \\
\hline & \multicolumn{3}{|c|}{$(\%)$} & & & \\
\hline $\mathrm{K}_{\mathrm{f}, \mathrm{ads}}$ & $0,78 *$ & $0,75 *$ & 0,10 & $0,61 *$ & $0,82 *$ & $-0,29$ \\
\hline \%Solução & 0,24 & 0,26 & $-0,97 *$ & 0,39 & $-0,56$ & $0,81 *$ \\
\hline $1 / \mathrm{n}_{\mathrm{ads}}$ & $0,63 *$ & $0,67 *$ & 0,07 & 0,52 & 0,60 & $-0,10$ \\
\hline $\mathrm{K}_{\text {foc }}$ & $-0,17$ & $-0,12$ & $0,73 *$ & $-0,34$ & 0,50 & $-0,61 *$ \\
\hline $\mathrm{pH}_{\mathrm{s}}$ & $-0,08$ & $-0,06$ & $-0,82 *$ & $-0,02$ & $-0,76^{*}$ & $0,92 *$ \\
\hline $\mathrm{K}_{\mathrm{f} \text {,des }}$ & $0,76^{*}$ & $0,68 *$ & 0,17 & 0,59 & $0,84 *$ & $-0,41$ \\
\hline $1 / \mathrm{n}_{\mathrm{des}}$ & $-0,04$ & $-0,05$ & $-0,35$ & 0,02 & $-0,39$ & 0,46 \\
\hline $\mathrm{C}_{\text {desorvida }}$ & $-0,27$ & $-0,25$ & $-0,81 *$ & $-0,14$ & $-0,89 *$ & $0,96 *$ \\
\hline \multicolumn{7}{|c|}{ Perfil LEr $(0-120 \mathrm{~cm})$} \\
\hline $\mathrm{K}_{\mathrm{f}, \mathrm{ads}}$ & $0,92 *$ & $0,78 *$ & $-0,78 *$ & $0,94 *$ & $0,75 *$ & $0,74 *$ \\
\hline \%Solução & 0,19 & 0,26 & $-0,28$ & $-0,04$ & $-0,25$ & 0,65 \\
\hline $1 / \mathrm{n}_{\mathrm{ads}}$ & $0,72 *$ & $0,76 *$ & $-0,77 *$ & 0,63 & 0,37 & $0,87 *$ \\
\hline$K_{\text {foc }}$ & $-0,06$ & 0,46 & $-0,47$ & 0,21 & $-0,06$ & 0,10 \\
\hline $\mathrm{pH}_{\mathrm{s}}$ & $0,74 *$ & 0,66 & $-0,68$ & 0,54 & 0,26 & 0,62 \\
\hline $\mathrm{K}_{\mathrm{f}, \mathrm{des}}$ & 0,59 & 0,25 & $-0,24$ & 0,39 & 0,47 & 0,50 \\
\hline $1 / n_{\text {des }}$ & 0,08 & $-0,02$ & 0,02 & $-0,16$ & $-0,18$ & 0,41 \\
\hline $\mathrm{C}_{\text {desorvida }}$ & $-0,28$ & $-0,23$ & 0,22 & $-0,48$ & $-0,52$ & 0,28 \\
\hline
\end{tabular}

Significativo a $5 \%$ de probabilidade; ${ }^{1 /}=\mathrm{pH}$ do sobrenadante.

significativo. A correlação positiva entre o $\mathrm{K}_{\mathrm{f} \text {.ads }}$ e a CTC deve-se à interação entre os sítios adsortivos, seja da matéria orgânica ou dos minerais de argila, e às moleculas do herbicida. Essa interação pode ocorrer por meio de pontes de hidrogênio e/ou por interações entre as moléculas com os cátions na superfície dos colóides.

Em se tratando dos Latossolos, os altos teores de silte (Tabela 1) podem ser explicados pela sua estrutura. Esses solos apresentam estrutura composta por microagregados formados por pequenos grânulos (Oliveira et al., 1992; Resende, 1985), que de alguma forma estão funcionando como silte e areia (Resende, 1985). Portanto, nesses solos, os teores de silte estão relacionados com a quantidade de microagregados que podem estar retendo o herbicida, impedindo-o de retornar à solução do solo, mesmo após agitação e centrifugação (Schwarzenbach et al., 1992), contribuindo assim para a obtenção de altos valores de $\mathrm{K}_{\mathrm{f} \text {,ads }}$. A redução na degradação do metsulfuronmetil e do chlorsulfuron com o tempo foi atribuída à migração desses herbicidas para dentro de "compartimentos" protegidos, onde não estavam disponíveis para os microrganismos (Walker et al., 1989).
Maiores valores de $\mathrm{pH}$ do sobrenadante acarretaram maior concentração do flazasulfuron em solução (\%solução), tanto no ensaio de sorção quanto no de dessorção (Tabela 4). Esses resultados estão de acordo com os obtidos por diversos autores (Gonzales \& Ukrainczyk, 1996; Ukrainczyk \& Rashid, 1995; Shea, 1986; Ukrainczyk \& Ajwa, 1996; Walker et al., 1989; Werkheiser \& Anderson, 1996), que também constataram correlação negativa entre a sorção de sulfoniluréias e o pH do sobrenadante. Ao contrário dos resultados obtidos neste trabalho, em estudos realizados por Oliveira Jr. et al. (2000) com sulfometuronmetil, nicosulfuron e metsulfuron-metil em solos brasileiros não se verificou correlação entre sorção e pH devido aos baixos valores de pH dos solos estudados.

$\mathrm{O}$ teor de $\mathrm{CO}$ reduziu com a profundidade nas amostras do perfil do LEr (Tabela 1). Tendência similar pode ser observada para os valores de $\mathrm{K}_{\text {f.ads }}$, à exceção da profundidade 5 (Tabela 3). Na amostra do LEr5, verifica-se acréscimo de aproximadamente $20 \%$ no valor de $\mathrm{K}_{\mathrm{f} \text {,ads }}$ e de mais de $70 \%$ no valor de $\mathrm{K}_{\text {foc }}$, em relação à profundidade anterior. Esse fato pode ser atribuído à redução do teor de matéria orgânica, com concomitante incremento no 
teor de argila natural verificada nessa profundidade. A importância do teor de argila natural nesta amostra também pode ser evidenciada pelo maior valor de $\mathrm{K}_{\text {foc }}$ calculado. A redução da sorção, portanto, da afinidade do flazasulfuron com o solo nas camadas mais profundas, indica maior risco de contaminação do lençol, caso o herbicida alcance camadas subsuperficiais.

Os valores de $\mathrm{K}_{\mathrm{f} \text {,ads }}$ correlacionaram-se positivamente com os teores de $\mathrm{CO}$, silte, CTC, umidade e $\mathrm{pH}$ e negativamente com os teores de argila quando a análise de correlação foi realizada com todas as amostras do perfil do LEr (Tabela 4). Ao contrário dos resultados descritos anteriormente e na literatura para diversas sulfoniluréias, o efeito do $\mathrm{pH}$ na retenção do flazasulfuron pelas amostras do LEr pode ser explicado pelos altos teores de minerais de carga dependentes de $\mathrm{pH}$ e pelos baixos teores de carbono orgânico dessas amostras. Esses minerais tendem a elevar o $\mathrm{pH}_{\mathrm{PCZ}}$ do solo (por exemplo: caulinita, gibbsita e óxidos de ferro que possuem altos valores de $\mathrm{pH}_{\mathrm{PCZ}}$ ), enquanto o $\mathrm{CO}$ tende a reduzir o $\mathrm{pH}_{\mathrm{PCZ}}$. Logo, essas amostras caracterizam-se por possuírem valor de $\mathrm{pH} \leq \mathrm{pH}_{\mathrm{PCZ}}$, portanto, com carga residual positiva. Por outro lado, aumentos de $\mathrm{pH}$ implicam aumentos na quantidade de moléculas aniônicas em solução $\left(\mathrm{pH} \geq \mathrm{pK}_{\mathrm{a}}\right)$, propiciando ligações catiônicas com os colóides do solo. Contribuição significativa dos óxidos de ferro e alumínio na sorção de herbicidas ácidos fracos tem sido observada em solos com baixos teores de carbono orgânico, conforme observado por Regitano et al. (1997, 2001, 2002), Ferreira et al. (2002), Rocha et al. (2002) e Koskinen \& Harper (1990). Oliveira Jr. et al. (2000) descreveram relação inversa entre sorção e $\mathrm{pK}_{\mathrm{a}}$ para sulfometuron-metil, nicosulfuron e metsulfuron-metil em solos brasileiros, indicando relação entre a especiação das moléculas e o pH. Discordando de Oliveira Jr., Sarmah et al. (2004) descreveram trabalhos em que a máxima sorção de herbicidas ácidos fracos foi observada quando os valores de $\mathrm{pH}$ do solo foram próximos dos valores de $\mathrm{pk}_{\mathrm{a}}$ do pesticida; no entanto, este máximo pode ser dependente de outras propriedades do solo.

$\mathrm{O}$ aumento nos teores de argila e a redução nos valores de $\mathrm{K}_{\mathrm{f} \text {,ads }}$ e $\mathrm{CO}$ com a profundidade explicam a correlação negativa entre os teores de argila e $\mathrm{K}_{\mathrm{f}, \text { ads }}$ nas amostras do LEr. Gonzales \& Ukrainczyk (1996) concluíram que a sorção do nicosulfuron nos solos brasileiros pela matéria orgânica foi mais significativa do que a adsorção pelos sítios dos minerais de carga dependentes de $\mathrm{pH}$.

Na Tabela 5, verifica-se que houve grande afinidade do flazasulfuron pelas amostras do perfil do LEr, principalmente nos menores valores de concentração inicial, em que só foi possivel detectar o herbicida na concentração de $0,8 \mathrm{mg} \mathrm{L}^{-1}$. A concentração média do herbicida no sobrenadante após o ensaio de sorção foi menor que $3,1 \%$, em todas as profundidades.

$\mathrm{O}$ menor valor de $\mathrm{K}_{\mathrm{f} \text {,ads }}$ para flazasulfuron foi observado no LE (Tabela 3), no valor de $\mathrm{pH}$ 6,6 do sobrenadante. Neste solo, a maior parte das moléculas do flazasulfuron $\left(\mathrm{pK}_{\mathrm{a}} 3\right.$ a 5) (Brown, 1990) deve ter permanecido na solução do solo, pois estariam na forma ionizada. Diversos autores (Sarmah et al., 2004; Ukrainczyk \& Rashid, 1995; Ukrainczyk \& Ajwa, 1996) verificaram sorção reduzida de herbicidas ionizáveis em solos com valores de $\mathrm{pH}$ maiores que os de $\mathrm{pK}_{\mathrm{a}}$. Além do alto valor de $\mathrm{pH}$ do sobrenadante, o solo LE possui o menor teor de matéria orgânica e argila, bem como alto teor de areia, comparado com outros solos (Tabela 1). Na Tabela 5, verifica-se que o solo LE foi o que apresentou maior porcentagem média $(32,5 \%)$ de flazasulfuron em solução após o ensaio de sorção. O fato anterior pode ser mais bem visualizado na Tabela 4, em que os aumentos nos valores de $\mathrm{pH}$ do sobrenadante implicam maior percentagem do flazasulfuron em solução (\% Sol), tanto no ensaio de sorção quanto no de dessorção $\left(\mathrm{C}_{\text {des }}\right)$.

Analisando a Tabela 5, observa-se que, nas amostras do LEr, a concentração do flazasulfuron em solução aumentou com o incremento da dose aplicada, caracterizando os valores de $1 / \mathrm{n}_{\text {ads }}$ menores que 1 (Tabela 3 ), o que mostra que pode ter havido saturação dos sítios de sorção pelo herbicida. Os valores de $1 / \mathrm{n}_{\text {ads }}$ variaram de 0,40 a 1,02 (Tabela 3 ). Os maiores valores de $1 / \mathrm{n}_{\text {ads }}$ foram obtidos nos solos que apresentaram maiores valores de $\mathrm{K}_{\mathrm{f} \text {,ads }}$, GH e LEr1, demonstrando linearidade entre o aumento da concentração do herbicida e sua afinidade pelas partículas do solo. À exceção desses dois solos, todos os valores de

Planta Daninha, Viçosa-MG, v. 23, n. 1, p. 101-113, 2005 
Tabela 5 - Porcentagem da concentração inicial do flazasulfuron que permaneceu em solução, em cada concentração, após ensaio de sorção e porcentagem média da concentração sorvida que permaneceu em solução após ensaio de dessorção $\left(\mathrm{C}_{\mathrm{des}}\right)$

\begin{tabular}{|c|c|c|c|c|c|c|c|c|}
\hline \multirow{3}{*}{ Solo } & \multicolumn{7}{|c|}{ Concentração inicial de flazasulfuron $\left(\mathrm{mg} \mathrm{L}^{-1}\right)$} & \multirow{2}{*}{$\left(\mathrm{C}_{\mathrm{des}}\right)$} \\
\hline & 0,07 & 0,2 & 0,8 & 1,5 & 3 & 5 & Média & \\
\hline & \multicolumn{8}{|c|}{ (\% em solução) } \\
\hline LE & 0,6 & 17,8 & 30,0 & 27,7 & 41,9 & 77,0 & 32,5 & 56,0 \\
\hline LRd & 6,6 & 5,4 & 13,6 & 13,8 & 14,3 & 20,2 & 12,3 & 8,8 \\
\hline GH & 16,4 & 15,3 & 26,3 & 16,8 & 17,9 & 24,5 & 19,5 & 1,3 \\
\hline LEr1 & 5,7 & 0,0 & 3,0 & 3,7 & 3,9 & 5,4 & 3,6 & 1,3 \\
\hline LEr2 & 0,0 & 0,0 & 2,9 & 2,8 & 2,8 & 4,4 & 2,2 & 1,1 \\
\hline LEr3 & 0,0 & 0,0 & 1,2 & 1,5 & 1,8 & 3,4 & 1,3 & 0,6 \\
\hline LEr4 & 0,0 & 0,0 & 0,0 & 0,6 & 1,5 & 2,7 & 0,8 & 0,4 \\
\hline LEr5 & 0,0 & 0,0 & 1,4 & 2,6 & 2,3 & 3,9 & 1,7 & 0,9 \\
\hline LEr6 & 0,0 & 0,0 & 2,2 & 1,4 & 2,3 & 5,4 & 1,9 & 1,0 \\
\hline LEr7 & 0,0 & 0,0 & 2,2 & 3,7 & 3,7 & 6,5 & 2,7 & 1,6 \\
\hline LEr8 & 0,0 & 0,0 & 3,0 & 5,8 & 5,6 & 8,0 & 3,7 & 3,1 \\
\hline
\end{tabular}

$1 / \mathrm{n}_{\text {ads }}$ encontrados estão abaixo de 0,90 evidenciando pouca dependência da sorção do herbicida com a sua concentração. Segundo Schwarzenbach et al. (1992), em geral, as isotermas de moléculas orgânicas ionizáveis são não-lineares. Gonzales \& Ukrainczyk (1996) atribuíram a não-linearidade da sorção do nicosulfuron, associada com valores de $1 / \mathrm{n}_{\text {ads }}<1$, à heterogeneidade nos sítios de sorção deste herbicida.

Os valores de $\mathrm{K}_{\text {foc }}$ apresentaram variação de 168,0 a $1.060,7$ nas amostras dos solos superficiais, indicando que, além do $\mathrm{CO}$, outras propriedades do solo também determinam a sorção do flazasulfuron (Gonzales \& Ukrainczyk, 1996). Analisando somente as amostras do LEr, o valor de $\mathrm{K}_{\mathrm{foc}}$ mostrou-se mais constante, exceto nas profundidades 4 e 8, que apresentaram os maiores teores de argila (Tabela 3).

Os parâmetros da isoterma de Freundlich ajustada para a dessorção do flazasulfuron encontram-se na Tabela 3. Os valores de $\mathrm{K}_{\mathrm{f} \text {,des }}$ variaram de 2,53 a 37,51. Observa-se que os valores de $\mathrm{K}_{\mathrm{f} \text {,des }}$ foram superiores aos do $\mathrm{K}_{\mathrm{f}, \text { ads }}$, ou seja, a afinidade do herbicida pelo solo foi maior no ensaio de dessorção. Werkheiser \& Anderson (1996) verificaram valores de $\mathrm{K}_{\mathrm{f}, \text { des }}$ cinco vezes maiores que $\mathrm{K}_{\mathrm{f} \text {,ads }}$ para primsulfuron em diferentes solos, inclusive amostras de horizonte B de Latossolos amostrados em Lavras-MG. Essa diferença entre os valores de $\mathrm{K}_{\mathrm{f}}$ dos ensaios de sorção e dessorção caracteriza o fenômeno denominado histerese (Koskinen et al., 1979; Koskinen \& Cheng, 1983; Pignatello, 1989; Koskinen \& Harper, 1990).

Analogamente ao ensaio de sorção, aumentos nos teores de $\mathrm{CO}$, silte e umidade acarretaram aumentos nos valores de $\mathrm{K}_{\mathrm{f} \text { des }}$ quando a análise de correlação foi feita com todos os solos em conjunto (Tabela 4). Quando a análise foi realizada sem o solo $\mathrm{GH}$, a correlação foi positiva e significativa para os teores de argila e umidade. Tanto a porcentagem do flazasulfuron em solução no ensaio de sorção quanto a concentração do herbicida dessorvida correlacionaram-se negativa e significativamente com o teor de argila, independentemente da presença do GH na análise conjunta de todos os solos. Isso porque as argilas foram importante fonte de acidez para a solução do solo, tanto no ensaio de sorção (Tabela 4) quanto no de dessorção (dado não apresentado), acarretando redução do pH do sobrenadante, tornando-o mais próximo ao $\mathrm{pK}_{\mathrm{a}}$ do herbicida. A sorção máxima de vários herbicidas tem ocorrido em valores de $\mathrm{pH}$ próximos do seu $\mathrm{pK}_{\mathrm{a}}$, conforme Mersie \& Foy (1985) descrevem para o chlorsulfuron. Maiores valores de $\mathrm{K}_{\mathrm{f}, \text { ads }}$ para nicosulfuron foram obtidos nos 
solos com maiores teores de carbono orgânico, e menores valores de $\mathrm{pH}$, em solos brasileiros de textura argila e de mineralogia semelhante entre si (Gonzales \& Ukrainczyk, 1996). Walker et al. (1989), estudando a sorção do chlorsulfuron e do metsulfuron-metil em solos amostrados em diferentes profundidades, obtiveram $\mathrm{K}_{\text {fads }}$ superiores a 10 para ambos os herbicidas somente para as amostras de solos com valores de $\mathrm{pH}$ próximos ao do $\mathrm{pK}_{\mathrm{a}}$ dos herbicidas e com altos teores de CO.

Considerando que no ensaio de dessorção foram utilizadas 48 horas de agitação, presume-se que o aumento observado na sorção no ensaio de dessorção possa ser atribuído a mudanças nas propriedades físicas e/ou químicas do sistema solo:solução, como a solubilização de matéria orgânica e a destruição dos agregados, resultando assim num aumento dos sítios sortivos (Koskinen \& Cheng, 1983). Savage \& Wauchope (1974) também atribuíram o aumento da adsorção do fluometuron pelo solo ao aumento da dispersão ou destruição dos microagregados do solo.

$\mathrm{O}$ efeito do $\mathrm{pH}$ do sobrenadante na retenção do flazasulfuron pode ser explicado, segundo Green \& Karickhoff (1990), pelo fato de o pH na superficie das argilas geralmente ser duas unidades menor que o $\mathrm{pH}$ da solução do solo. Como a solubilidade do flazasulfuron é relativamente baixa (Figura 1), tendendo a diminuir a menores valores de $\mathrm{pH}$ (Smith, 1995), a queda no valor de $\mathrm{pH}$ do sobrenadante pode ter contribuído para que a grande maioria das moléculas tenha permanecido na forma molecular, próximo ou na superficie das argilas, pois a relação entre as espécies iônica e molecular é de 1:1 quando o $\mathrm{pH}$ se aproxima do $\mathrm{pK}_{\mathrm{a}}$ do herbicida. Dessa forma, é de se esperar maior partição com o carbono orgânico do solo ou mesmo precipitação do herbicida na superficie dos minerais.

Por outro lado, os baixos valores de $\mathrm{pH}$ do sobrenadante também podem ter acarretado hidrólise do flazasulfuron, contribuindo para subestimar a concentração de equilíbrio do herbicida no ensaio de dessorção e, conseqüentemente, os valores de $\mathrm{K}_{\mathrm{f} \text {,ads }}$ (Havens et al., 1995). Walker et al. (1989) verificaram maior taxa de degradação dos herbicidas chlorsulfuron e metsulfuron-metil nas amostras dos solos com menores valores de $\mathrm{pH}$.
Estudos realizados por Sabadie $(1990,1991)$ e Sabadie e Bastide (1990) evidenciaram maior hidrólise de sulfoniluréias em solução na presença de argilas ou ácidos húmicos em suspensão e cátions de metais, como $\mathrm{Fe}^{+3}$. Logo, para os herbicidas sulfoniluréias é importante a atenção aos picos do HPLC de outras substâncias que não correspondam à substância-padrão, que provavelmente sejam metabólitos.

À exceção do LEr8, todos os valores de $1 / \mathrm{n}_{\text {des }}$ foram menores que 1,0 (Tabela 3 ). A reversibilidade da dessorção pode ser avaliada pela mudança no valor do $\mathrm{K}_{\mathrm{f}}$ do ensaio de sorção e dessorção e pela razão $\mathrm{n}_{\text {des }} / \mathrm{n}_{\text {ads }}$ (van Genuchten et al., 1974; Pignatello, 1989; Gonzales \& Ukrainczyk, 1996). A sorção é dita reversivel quando há sobreposição da curva no ensaio de sorção e dessorção, ou seja, quando $\mathrm{K}_{\mathrm{f} \text {,des }} \approx \mathrm{K}_{\mathrm{f} \text {,ads }}$ e $\mathrm{n}_{\text {des }} \approx \mathrm{n}_{\text {ads }}$. A não-reversibilidade indicativa do fenômeno de histerese ocorre quando não há sobreposição das isotermas de sorção e dessorção, sendo muito comum para as substâncias orgânicas no solo (Pignatello, 1989; Gonzales \& Ukrainczyk, 1996; Koskinen \& Harper, 1990). A histerese positiva, em que $\mathrm{K}_{\mathrm{f} \text {,des }}>\mathrm{K}_{\mathrm{f}, \mathrm{dds}}$ e $\mathrm{n}_{\text {des }}<\mathrm{n}_{\text {ads }}$, observada por Gonzales \& Ukrainczyk (1996) na dessorção do nicosulfuron em solos brasileiros pode ser observada para flazasulfuron em todos os solos estudados, com exceção do LE, LRd e LEr8. Além da maior interação do flazasulfuron pelos colóides do solo no ensaio de dessorção por meio de interações de superficie, como pontes de hidrogênio, forças de van der Waals e quimissorção, interações hidrofóbicas também podem estar contribuindo para o fenômeno de histerese positiva.

A hidrólise do flazasulfuron seguiu modelo de primeira ordem, sendo maior nos menores valores de $\mathrm{pH}$ e em maiores valores de temperatura. A sorção desse herbicida apresentou correlação positiva e significativa com os teores de carbono orgânico, silte, CTC e umidade do solo. Maiores valores de $\mathrm{pH}$ do solo reduziram a sorção do flazasulfuron, indicando maior risco de lixiviação. No caso das amostras do perfil do LEr, o teor de argila correlacionou-se negativamente com a sorção do flazasulfuron, enquanto incrementos nos valores de $\mathrm{pH}$ aumentaram-na.

Planta Daninha, Viçosa-MG, v. 23, n. 1, p. 101-113, 2005 


\section{AGRADECIMENTOS}

À UENF, Embrapa e Fapemig, pelo auxílio financeiro; à Companhia ISK Bioscience, pelo produto técnico; e ao laboratorista Nilson Machado Lopes e aos bolsistas Tricia Cristina Lessa de Faria, Gibson Antônio Santos Soares e Roqueline Rodrigues Silva, pela ajuda.

\section{LITERATURA CITADA}

BAILEY, C. W.; WHITE, J. L. Factors influencing the adsorption, desorption and movement of pesticides in soil. In: RESIDUE review the triazine herbicides. New York: Springer Verlag. 1970. v. 32. p. 29-92.

BERTRAND, C. et al. Flazasulfuron alcoholysis, chemical hydrolysis, and degradation on various minerals. J. Agric. Food Chemistry, v. 51, p. 7717-7721, 2003.

BERGLOF, T. et al. Metsulfuron methyl sorptiondesorption in field -moist soils. J. Agric. Food Chemistry, v. 51, p. 3598-3603, 2003.

BLAIR, A. M.; MARTIN, T. D. A review of the activity fate and mode of action of sulfonylurea herbicides. Pest. Sci., v. 22, p. $195-219,1988$.

BROWN, H. M. Mode of action, crop selectivity, and soil relations of the sulfonylurea herbicides. Pest. Sci., v. 29, n. 3, p. 263-281, 1990.

\section{EMPRESA BRASILEIRA DE PESQUISA} AGROPECUÁRIA - EMBRAPA. Manual de métodos de análise de solos. 2.ed. Rio de Janeiro: 1997. 212 p.

FELDING, G. Pesticide adsorption as a function of depth below surface. Pest. Sci., v. 50, p. 64-66, 1997.

FERREIRA, J. A. et al. Sorption interactions between Imazaquin and a humic acid extracted from a typical brazilian oxisol. J. Environ. Quality, v. 31, p. 1665-1670, 2002.

GONZALES, J. M.; UKRAINCZYK, L. Adsorption and desorption of nicosulfuron in soils. J. Environ. Qual., v. 25, p. 186-1192, 1996.

GREEN, R. E., KARICKHOFF, S. W. Sorption estimates for modeling. In: CHENG, H. H. (Ed.) Pesticides in the soil environment: processes, impacts, and modeling. Madison: Soil Science Society of America, 1990. p. 79-101.

HARPER, S. S. Sorption of metribuzin in surface and subsurface soils of the Mississippi Delta Region. Weed Sci. v. 36, p. $84-89,1988$.

HASHIZUME, B. New pesticide flazasulfuron, a new turf herbicide (Shibagen ${ }^{\circledR}$ ). Japan Pest. Inf., v. 57, p. 27-30, 1990.

Planta Daninha, Viçosa-MG, v. 23, n. 1, p. 101-113, 2005
HAY, J. V. Chemistry of sulfonylurea herbicides. Pestic. Sci., v. 29, n. 3, p. 247-261, 1990.

HAVENS, P. L.; SIMS, G. K.; ERHARDT-ZABIK, S. Fate of herbicides in the environment. In: SMITH, A. E. (Ed.)

Handbook of weed management systems. New: M. Dekker, 1995. p. 245-278.

HYUN, S.; LEE, L. S. Factors controlling sorption of prosulfuron by variable-charge soils and model sorbents. J. Environ. Quality, v. 33, p. 1354-1361, 2004.

HYUN, S.; LEE, L. S.; RAO, P.S.C. Significance of anion exchange in pentachlorophenol sorption by variable-charge soils. J. Environ. Quality, v. 32, p. 966-976, 2003.

JENKS, B. M. et al. Influence of surface and subsurface soil properties on atrazine sorption and degradation. Weed Sci., v. 46, p. 132-138, 1998.

KOSKINEN, W. C.; CHENG, H. H. Effects of experimental variables on 2,4,5-T adsorption-dessorption in soil. J. Environ. Quality, v. 12, n. 3, p. 325-330, 1983.

KOSKINEN, W. C.; HARPER, S. S. The retention process: mechanisms. In: CHENG, H. H. (Ed.) Pesticides in the soil environment: processes, impacts, and modeling. Madison: Soil Science Society of America, 1990. p. 51-77.

KOSKINEN, W. C.; O'CONNOR, G. A.; CHENG, H. H. Characterization of hysteresis in the desorption of 2,4,5-T from soil. Soil Sci. Soc. Am. J., v. 43, p. 871-874, 1979.

MALIK, N.; DRENNAN, D. S. H. Adsorption-desorption equilibria of carbon-14 labeled fluridone at low solution concentrations and soil water ratios. Can. J. Soil Sci., v. 69, p. $567-578,1989$.

MERSIE, W.; FOY, C. L. Phytotoxicity and adsorption of chlorsulfuron as affected by soil properties. Weed Sci., v. 33 , n. 4 , p. $564-568,1985$.

MEYER, M. T.; THURMAN, E. M. Herbicide metabolites in surface water and groundwater. Washington: American Chemical Society, 1995. 318 p. (Symposium Series, 630).

OLIVEIRA, J. B.; JACOMINE, P. K. T.; CAMARGO, M. N. Classes gerais de solos do Brasil: guia auxiliar para seu reconhecimento. 2 ed. Jaboticabal: FUNEP, 1992. $201 \mathrm{p}$.

OLIVEIRA Jr., R.S.; KOSKINEN, W.C.; FERREIRA, F.A. Sorption and leaching potential of herbicides on Brazilian soils. Weed Res., v. 41, p. 97-110, 2000.

OLIVEIRA, M. F. Adsorção, lixiviação e persistência de flumioxazin e metribuzin em diferentes solos. 1995. 71 f. Dissertação (Mestrado em Fitotecnia) - Universidade Federal de Viçosa, Viçosa, 1995. 
PIGNATELLO, J. J. Sorption dynamics of organic compounds in soils and sediments. In: SOIL SCIENCE COCIETY OF AMERICA (Ed.) Reactions and movement of organic chemicals in soils. Madison: 1989. p. 45-80. (Special Publication Number, 22)

RACKE, K. D. Pesticide fate in tropical soils. In: WORKSHOP ON PESTICIDES, USES AND ENVIRONMENTAL SAFETY IN LATIN AMERICA, 1996, São Paulo. Book of Abstracts... São Paulo: Sociedade Brasileira de Química, 1996. p. 15

REGITANO, J. B. et al. Retention of imazaquin in soil. Environ. Toxic. Chem., v. 16, n. 3, p. 397-404, 1997.

REGITANO, J. B.; ALLEONI, L. R. F.; TORNISIELO, V. L. Atributos de solos tropicais e a sorção de imzaquin. Sci. Agric., v. 58, n. 4, p. 801-807, 2001.

REGITANO, J. B. et al. Imazaquin mobility in tropical soils in relation to soil moisture and rainfall timing. Weed Res., v. 42, p. 271-279, 2002.

RESENDE, M. Aplicações de conhecimentos pedológicos à conservação de solos. Inf. Agropec., v. 11, n. 128, p. 3-18, 1985.

RHODES, R. C.; BELASCO, I. J.; PEASE, H. L. Determination of mobility and adsorption of agrochemicals on soils. J. Agric. Food Chem., v. 18, n. 3, p. 524-528, 1970.

ROCHA, W. S. D. et al. Sorption of imazaquin in soils with positive balance of charges. Chemosphere, v. 49, p. 263270, 2002.

RODRIGUES, B. N.; ALMEIDA, F. S. Guia de herbicidas. 4. ed. Londrina: Instituto Agronômico do Paraná, 1998. $648 \mathrm{p}$.

ROOWELL, D. L. Soil science: methods and applications. London: Longman Scientistic Technical, 1994. 350 p.

SABADIE, J. Hydrolyse chimique acide du metsulfuron méthyle. Weed Res., v. 30, n. 6, p. 413-419, 1990.

SABADIE, J. Alcoolyse et hydrolyse chimique acide du chlorsulfuron. Weed Res., v. 31, n. 5, p. 309-31, 1991.

SABADIE, J.; BASTIDE, J. Dégradation du metsulfuron méthyle déposé sur divers supports minéraux. Weed Res., v. 30, n. 1, p. 1-8, 1990.

SARMAH, A. K.; MULLER, K.; AHMAD, R. Fate and behaviour of pesticides in the agroecosystem - a review with a New Zealand perspective. Aust. J. Soil Res., v. 42, p. 125-154, 2004.

SARMAH, A. K.; SABADIE, J. Hydrolysis of sulfonylurea herbicides in soils and aqueous solutions; a review. J. Agric. Food Chem., v. 50, p. 6253-6265, 2002.
SAVAGE, K. E.; WAUCHOPE, R. D. Fluometuron adsorption-desorption equilibria in soil. Weed Sci., v. 22, n. 2, p. 106-110, 1974.

SCHROEDER, J. Chlorimuron and imazaquin persistence in selected southern soil under controlled conditions. Weed Sci., v. 42, p. 635-640, 1994.

SCHWARZENBACH, R. P.; GSCHWEND, P. M.; IMBODEN, D. M. Environmental organic chemistry. New York: John Wiley \& Sons, 1992. 657 p.

SHEA, P. J. Chlorsulfuron dissociation and adsorption on selected adsorbents and soils. Weed Sci., v. 34, n. 3, p. 474478, 1986.

SMITH, A. E. Review of analytical methods for sulfonylurea herbicides in soil. J. Environ. Anal. Chem., v. 59, p. 97-106, 1995.

SMITH, A. E.; AUBIN, A. J. Degradation of $\left[{ }^{14} \mathrm{C}\right]$ amidosulfuron in aqueous buffers and in an acidic soil. J. Agric. Food. Chem., v. 41, n. 12, p. 2400-2403, 1993.

STORK, P. R. Field leaching and degradation of soil applied herbicides in a gradationally texture alkaline soil: chlorsulfuron and triasulfuron. Austr. J. Agric. Res., v. 46, p. 1445-1458, 1995.

THIRUNARAYANAN, K.; ZIMDAHL, R. L.; SMIKA, D. E. Chlorsulfuron adsorption and degradation in soil. Weed Sci., v. 33, p. 558-563, 1985.

UKRAINCZYK, L.; AJWA, H. A. Primisulfuron sportion on minerals and soils. Soil Sci. Soc. Am. J., v. 60, p. 460-467, 1996.

UKRAINCZYK, L.; RASHID, N. Irreversible sorption of nicosulfuron on clay minerals. J. Agric. Food. Chem., v. 43, n. 4, p. 855-857, 1995.

Van GENUCHTEN, M. T. H.; DAVIDSON, J. M.; WIERENGA, P. J. An evaluation of kinetic and equilibrium equations for the prediction of pesticide movement through porous media. Soil Sci. Soc. Am. Proc., v. 38, p. 29-35, 1974.

VICARI, A. et al. Primisulfuron and rimsulfuron degradation in aqueous solution and adsorption in six Colorado soils. Weed Sci., v. 44, p. 672-677, 1996.

WALKER, A.; COTTERILL, E. G.; WELCH, S. J. Adsorption and degradation of chlorsulfuron and metsulfuron-methyl in soils from different depths. Weed Res., v. 29, p. 281-287, 1989.

WERKHEISER, W. O.; ANDERSON, S. Organic chemicals in the environment. J. Environ. Qual., v. 25, n. 4, p. 809814, 1996.

Planta Daninha, Viçosa-MG, v. 23, n. 1,p. 101-113, 2005 